Molecular Science

June 2016

\title{
More effective chemotherapy drugs? Studying the role of human equilibrative nucleoside transporter 1 (hENT1)
}

How could we make nucleoside analog (NA) drugs - which are widely used chemotherapy - have a greater therapeutic effect? This was the question that drove a compelling investigation recently undertaken by researchers in Ryerson's Department of Chemistry and Biology.

Encouraged by her supervisor Dr. Imogen Coe, Maliha Zafar, MSc, a recent graduate from the Molecular Science Graduate Program, placed her focus on the human equilibrative nucleoside transporter 1 (hENT1).

Why this transporter in particular? In answering, Zafar pauses to explain the role of nucleoside transporters. "A specialized family of proteins, called the nucleoside transporters (NTs), sits in the plasma membrane and facilitates the entry of naturally-occurring nucleosides and NA drugs into the cells by acting as gates that open and close, specifically for these compounds." Zafar adds that hENT1 is a key drug transporter. "hENT1 is a major route of entry of nucleosides and nucleoside analog drugs." And yet, despite its clinical importance, she says the regulation of hENT1 is still poorly understood.

Supervised by Dr. Coe, Zafar and fellow researcher Zlatina Tarmakova made a curious discovery. Initial work seemed to suggest lessening drug efficacy over time, says Zafar. Research showed, for example, that the proteins responsible for allowing the drug into the cell, had been internalized by cells away from the plasma membrane, she says.

Yet in a fascinating twist, Zafar says, "I also found that some ENT1 proteins remain at the plasma membrane and continue to allow drug to enter. My work suggests that hENT1 proteins exist as two sub-populations in the plasma membrane, inactive and active [and] that prolonged exposure to a drug leads to the internalization of the inactive population of hENT1 proteins only, which results in an overall decrease in the abundance of hENT1 at the plasma membrane, but leaves behind active hENT1. So there is no change in overall drug uptake through hENT1."

Next, the Ryerson team explored potential practical applications. "Following these findings," says Zafar, "I determined if pre-treating cells with a nucleoside would change the subsequent toxicity of gemcitabine (a NA drug used in the treatment of pancreatic cancer). To our surprise, we found that cytidine pre-treatment followed by gemcitabine treatment led to an increase in the number of dead human pancreatic cancer cells compared to gemcitabine alone."

Ultimately, she says, "Our findings suggest that exposing hENT1 to its substrate (a compound that it transports routinely) leads to some hENT1 proteins becoming "exhausted" (inactive) and being recycled away from the plasma membrane - leading to an increased proportion of "primed" or active hENT1 proteins which can take up more drug and thereby achieving a greater therapeutic effect from a smaller dose of drug."

Ryerson researchers recently documented their findings in a paper called "Extended exposure to substrate regulates the human equilibrative nucleoside transporter 1 (hENT1)" to be published in the journal Nucleosides, Nucleotides and Nucleic Acids. 
Since graduating from Ryerson in the fall of 2015, Zafar has joined vaccine maker Sanofi Pasteur in Toronto as quality operations manager. She credits her time at Ryerson education as a key part of her professional success.

"The Molecular Science program at Ryerson University allowed my curious mind to explore cell biology in a competitive, yet friendly environment. Dr. Coe and other Ryerson faculty members continually provided constructive criticism. I also had tremendous support from our research associates, Dr. Zlatina Tarmakova and Dr. Natalia Grane Boladeras, and also Christopher Spring from St. Michael's Hospital Research Core Facilities."

Zafar's research was funded by Dr. Coe's Natural Science and Engineering Research Council (NSERC) grant. Zafar also received a Ryerson Graduate Scholarship and Canada Graduate Scholarship from Canadian Institutes of Health Research (ClHR). 